\title{
CONTROLLED ISLANDING OF POWER SYSTEMS CONSIDERING VOLTAGE STABILITY CONSTRAINTS
}

\author{
Panayiotis Demetriou $^{1 *}$, Alexis Kyriacou ${ }^{1}$, Elias Kyriakides ${ }^{1}$, and Christos Panayiotou ${ }^{1}$ \\ ${ }^{1}$ Department of Electrical and Computer Engineering and KIOS Research and Innovation Center of Excellence, \\ University of Cyprus, Nicosia, Cyprus \\ *demetriou.k.panayiotis@ucy.ac.cy
}

\begin{abstract}
Keywords: BLACKOUT PREVENTION, INTENTIONAL CONTROLLED ISLANDING, GENERATOR COHERENCY, MILP FORMULATION, VOLTAGE STABILITY.
\end{abstract}

\begin{abstract}
An Intentional Controlled Islanding (ICI) algorithm that directly determines an islanding solution with minimal power-flow disruption for any given number of islands, while ensuring that each island contains only coherent generators was previously proposed and tested using IEEE test systems. The proposed algorithm is based on an exact Mixed Integer Linear Programming Formulation (MILP). However, since voltage instability has been found to be one of the main causes of several recent blackouts in the world, in this paper, the aforementioned algorithm is extended to consider voltage stability constraints. The extended ICI algorithm guarantees sufficient generation capacity (active and reactive power) to match the load consumption within each island. At the same time, it reduces the possibility of voltage collapse by assigning the weakest buses to the islands in such a way to be as close as possible to the sources or sinks of reactive power. The new constraints are expected to further improve the transient stability of the formed islands.
\end{abstract}

\section{Introduction}

Recent power system blackouts have been characterized by a progressive and uncontrollable decrease in voltage magnitude at the system buses. These voltage instability phenomena are termed "voltage collapse". Voltage collapse is typically associated with reactive power demand of load not being met due to shortage in reactive power production and transmission.

Intentional controlled islanding (ICI) has been proposed as an effective corrective control action to mitigate the occurrence and consequences of large-scale blackouts in power systems [1]. When the system is subject to a severe disturbance and the conventional control systems are unable to keep the system stable, ICI can determine in real-time a set of lines to be disconnected across the transmission system to create stable and sustainable subsystems (also known as islands), while maintaining generator coherencies and other static and dynamic constraints [2], [3].

In the literature, several approaches that determine possible splitting strategies are reported. In these approaches, ICI is usually modelled as a constrained combinatorial optimization, whose two main types of objective function are minimal power-flow disruption and minimal power imbalance within islands [4-8]. Nonetheless, the concept of controlled islanding strategies that consider voltage stability constraints is still an unexplored research area.

In [5], the authors have proposed an exact ICI algorithm for solving effectively the ICI problem. The proposed algorithm directly determines an islanding solution with minimal power-flow disruption for any given number of islands, while ensuring that each island contains only coherent generators. Moreover, the algorithm enables operators to constrain any line to be excluded from the solution, ensures the connectivity inside the islands and allows the control of their size.

In this paper, the aforementioned algorithm is extended to consider voltage stability constraints. The extended ICI algorithm guarantees sufficient generation capacity (active and reactive power) to match the load consumption within each island. At the same time, it reduces the possibility of voltage collapse by assigning the weakest buses to the islands in such a way to be electrically as close as possible to the sources or sinks of reactive power (e.g., generators, shunt capacitors, storage systems). The weakest buses are the first points where voltage collapse appears in a severe contingency. This paper identifies the weakest buses in the power system by utilizing an existing line stability index. The new constraints are expected to further improve the transient stability of the formed islands. This paper is organized as follows: the existing MILP ICI is presented in Section 2. The additional voltage stability constraints incorporated to the MILP formulation are explained in Section 3. Section 4 presents the simulation results for the IEEE 39-bus test system. The paper concludes in Section 5.

\section{Intentional Controlled Islanding}

This section presents the existing MILP ICI algorithm for minimal power-flow disruption. The graph theory fundamentals are also given in this section.

\subsection{ICI Problem Modeling}

In graph theory, an undirected graph-model $\mathcal{G}=(\mathcal{V}, \mathcal{E})$ can be used to describe an $m$-generator and $n$ bus power system. In this graph-model, the node set 
$\mathcal{V}=\left\{v_{1}, \ldots, v_{n}\right\}$ denotes the buses while the edge set $\mathcal{E}$ with elements $e_{i, j}(i, j=1, \ldots, n)$ denotes the transmission lines. The set $\mathcal{V}^{\text {gen }}$ is a subset of the node set $\mathcal{V}$ that contains only those buses with generators directly connected to them. The set $\mathcal{W}$, with elements $w_{i, j}(i, j=1, \ldots, n)$, is a set of edge weights representing the weight factors (power flow) associated with the transmission lines.

A cutset $\mathcal{E}_{\mathcal{S}} \subset \mathcal{E}$ [9] is the set of edges to be removed to split $\mathcal{G}$ into $K \in \mathbb{Z}^{+}$subgraphs $\mathcal{G}_{k}=\left(\mathcal{V}_{k}, \mathcal{E}_{k}\right)$, where $k \in \mathcal{K}$, $\mathcal{K}=\{1, \ldots, K\}$. For $K$ islands, the sum of the edge weights within the cutset $\mathcal{E}_{\mathcal{S}}$ is called the cut, which is defined as,

$$
\operatorname{cut}\left(\mathcal{V}_{h}, \mathcal{V}_{k}\right)=\sum_{i \in \mathcal{V}_{h}, j \in \mathcal{V}_{k}} w_{i, j}, h \neq k, h, k \in \mathcal{K}
$$

\subsection{Exact ICI Algorithm for Minimal Power-Flow Disruption}

The existing MILP ICI algorithm proposed in [5] solves the ICI problem for minimal power-flow disruption by representing it as a constrained combinatorial optimization,

$$
\min \left(\sum_{\substack{i \in \mathcal{V}_{h}, j \in \mathcal{V}_{k} \\ h \neq k, h, k \in \mathcal{K}}} \frac{\left|P_{i, j}\right|+\left|P_{j, i}\right|}{2}\right) \text { s.t. } \mathcal{V}_{k}^{\text {gen }} \subset \mathcal{V}_{k}, \mathcal{E}_{C} \subset \mathcal{E}
$$

In this framework, the node subset $\mathcal{V}_{k}^{\text {gen }}$ contains the coherent generator groups for each island $\mathcal{G}_{k}$, while the edge subset $\mathcal{E}_{C}$ contains all the branches that cannot be disconnected. $P_{i, j}$ and $P_{j, i}$ represent the active power flow in the transmission line from bus $i$ to $j$, and from $j$ to $i$, respectively.

2.2.1 MILP formulation to solve the ICI problem [5]: Consider the graph representation of the power system described in Section II-A. The goal of the MILP formulation is to partition the graph into $K$ subgraphs indicating the islands while a) minimizing the weight of the edges that are not included in any subgraph which is defined as the partitioning cost and is described by the objective function:

$$
\text { Partitioning Cost }=\min _{z} \sum_{(i, j) \in \mathcal{E}} \frac{1}{2}\left(1-z_{i, j}\right) w_{i, j}
$$

b) controlling the size of subgraphs and c) ensuring that each produced subgraph is connected. In addition, the resulting subgraphs $\mathcal{G}_{k}=\left(\mathcal{V}_{k}, \mathcal{E}_{k}\right)$ where $\bigcup_{k} \mathcal{V}_{k}=\mathcal{V}, k \in \mathcal{K}$ must follow a minimum cardinality restriction as $\left|\mathcal{V}_{k}\right| \geq M, k \in \mathcal{K}$, where $M \in \mathbb{N}^{+}$. Variables $z_{i, j},(i, j) \in \mathcal{E}$ are defined as the decision variables where $z_{i, j}=1$ if the edge is included in any subgraph and $z_{i, j}=0$ otherwise. It is noted that a detailed description of the aforementioned MILP formulation can be found in [10].

2.2.2 Preprocessing procedure: The first step of the existing MILP ICI algorithm is a preprocessing procedure, which is applied on graph $\mathcal{G}$ in order to reduce the search space of the
MILP and the overall complexity of the problem [5]. The objective of the preprocessing procedure is to find the tree $\mathcal{T}_{k}=\left(\mathcal{V}_{k}^{T}, \mathcal{E}_{k}^{T}\right), \quad k \in \mathcal{K}$ in $\mathcal{G}$ that connects all the generator nodes of each coherent group $\mathcal{V}_{k}^{\text {gen }}$ with the minimum number of nodes. The nodes that are included in each $\mathcal{T}_{k}$ are then directly assigned to their resulting island in the MILP formulation, ensuring the generator coherency. Moreover, it is important to mention that the preprocessing procedure ensures the presence of at least one load-bus in each $\mathcal{T}_{k}$ to avoid the production of isolated generator nodes, and consequently, to form more reliable islands.

\section{Controlled Islanding and Voltage Stability}

In this section, the controlled islanding and the voltage stability are combined under the same framework. This is achieved though the incorporation of additional voltage stability constraints to the MILP formulation presented in Section 2.

\subsection{Sufficient generation capacity (active \& reactive power)}

Consider the node sets $\mathcal{V}_{k}^{\text {gen }}=\left\{v_{1}, \ldots, v_{n_{k}^{\text {gen }}}\right\}$ and $\mathcal{V}_{k}^{\text {load }}=\left\{v_{1}, \ldots, v_{n_{k} \text { load }}\right\}$ which denote the generator nodes and load nodes in each island $\mathcal{G}_{k}$ respectively, where $n_{k}^{\text {gen }}$ denotes the number of generators and $n_{k}^{\text {load }}$ the number of loads in island $\mathcal{G}_{k}$. Furthermore, consider a mapping function Fgen $_{k}:\{1, \ldots, n\} \rightarrow\left\{1, \ldots, n_{k}^{\text {gen }}\right\}$ for the generator nodes and its inverse function $\operatorname{Fgen}_{k}^{-1}:\left\{1, \ldots, n_{k}^{\text {gen }}\right\} \rightarrow\{1, \ldots, n\}$ satisfying:

$$
\begin{aligned}
& \operatorname{Fgen}_{k}(i)=\left\{j: v_{j}=v_{i}, i \in\{1, \ldots, n\}, j \in\left\{1, \ldots, n_{k}^{\text {gen }}\right\}\right\}, \\
& \operatorname{Fgen}_{k}^{-1}(j)=\left\{i: v_{i}=v_{j}, i \in\{1, \ldots, n\}, j \in\left\{1, \ldots, n_{k}^{\text {gen }}\right\}\right\}
\end{aligned}
$$

Note that a similar mapping function is considered for the load nodes. The constraint of sufficient generation capacity is as follows:

$$
\begin{gathered}
\sum_{i \in\left\{1, \ldots, n_{k}^{\text {gen }}\right\}} \text { Pgen }_{i, k}^{\max } \geq \sum_{i \in\left\{1, \ldots, n_{k}^{\text {load }}\right\}} \text { Pload }_{i, k} \geq \sum_{i \in\left\{1, \ldots, n_{k}^{\text {gen }}\right\}} \text { Pgen }_{i, k}^{\min }, \\
\sum_{i \in\left\{1, \ldots, n_{k}^{\text {gen }}\right\}} \text { Qgen }_{i, k}^{\max } \geq \sum_{i \in\left\{1, \ldots, n_{k}^{\text {load }}\right\}} \text { Qload }_{i, k} \geq \sum_{i \in\left\{1, \ldots, n_{k}^{\text {gen }}\right\}} \text { Qgen }_{i, k}^{\min }
\end{gathered}
$$

where Pgen $_{i, k}$, Qgen $_{i, k}$ and Pload $_{i, k}$, Qload $_{i, k}$ are the generation and load consumption of bus $i$ in island $k$ respectively. Assuming that the generators are able to operate at full rated power, this constraint guarantees sufficient generation capacity to match the active and reactive power demand in the formed islands. At the same time, it avoids the unnecessary shut down of generators just to achieve the load-generation balance.

\subsection{Voltage collapse prevention}

The weakest buses are defined as the buses which lack reactive power support the most in order to defend against voltage collapse. In this paper, the weakest buses are identified by utilizing the Fast Voltage Stability Index (FVSI) [11]. For a typical transmission line, the FVSI is calculated by 


$$
F V S I=\frac{4 Z^{2} Q_{r}}{V_{s}^{2} X}
$$

where $Z$ is the line impedance, $X$ is the line reactance, $Q_{r}$ is the reactive power at the receiving end, and $V_{s}$ is the sending end voltage. The FVSI must be below 1.0 for a stable transmission line. If FVSI goes beyond 1.0, one of the buses that is connected to the line will experience a sudden voltage drop (i.e., it is a weak bus) leading to system collapse.

The voltage collapse prevention constraint assigns the weakest buses to the islands in such a way to be as close as possible to the reactive power sources by utilizing the electrical distance concept. The electrical distance which corresponds to the Thevenin equivalent between the bus $i$ and bus $j$ is calculated as [12]:

$$
r_{i, j}=|B|_{i, i}^{+}+|B|_{j, j}^{+}-|B|_{i, j}^{+}-|B|_{j, i}^{+}, \quad i, j \in \mathcal{V}
$$

This adjustment maximizes the voltage increase, and thus, it improves the system voltage stability.

\section{Simulation Results}

The effectiveness of the aforementioned extended ICI algorithm is demonstrated using the dynamic model of the IEEE 39-bus test system (Fig. 1) [13]. This system has 10 synchronous generators, 34 transmission lines, 12 transformers and 19 constant power loads. The algorithm is aimed to be used following the determination of the necessity to split the power system. All times quoted are based upon simulations performed in Matlab (on a PC with $3.10 \mathrm{GHz}$ dual core CPU and 4 GB RAM).

\subsection{Case Study 1}

Testing case description: At time $t=1 \mathrm{~s}$, a three phase to ground fault occurs at bus 2 and is cleared $0.65 \mathrm{~s}$ later (at $t=$ $1.65 \mathrm{~s}$ ). The swing curves of the generators during this fault are shown in Fig. 2. As it can be seen, shortly after the clearance of the fault, three groups of generators are formed: $\{\mathrm{G} 31, \mathrm{G} 32\},\{\mathrm{G} 33, \mathrm{G} 34, \mathrm{G} 35, \mathrm{G} 36\}$, and $\{\mathrm{G} 30, \mathrm{G} 37, \mathrm{G} 38$, G39\}. Moreover, as Fig. 2 depicts, if the system is not split into three Islands, the speed of the generators increases and their terminal voltages significantly reduce. Thus, a blackout is unavoidable even if the fault is removed. In this case, the necessity to separate the system is assumed to be at $1.7 \mathrm{~s}$. Hence, considering the power flow and actual topology of the system at $\mathrm{t}=1.7 \mathrm{~s}$, the proposed extended ICI algorithm is used to find the optimal islanding solution.

The first step of the algorithm is the preprocessing procedure, which finds first the trees that connect all the generators of each coherent group with the minimum number of nodes (Fig. 3). As mentioned in Section 2, the nodes of these trees are then served as an additional constraint to the MILP formulation. At the same time, the weakest buses in the power system are identified by calculating the FVSI index of each transmission line. For this case study, ten weak buses are identified, i.e., bus 2, 3, 4, 16, 18, 19, 25, 26, 28, and 29 (shaded buses in Fig. 4). According to the voltage collapse

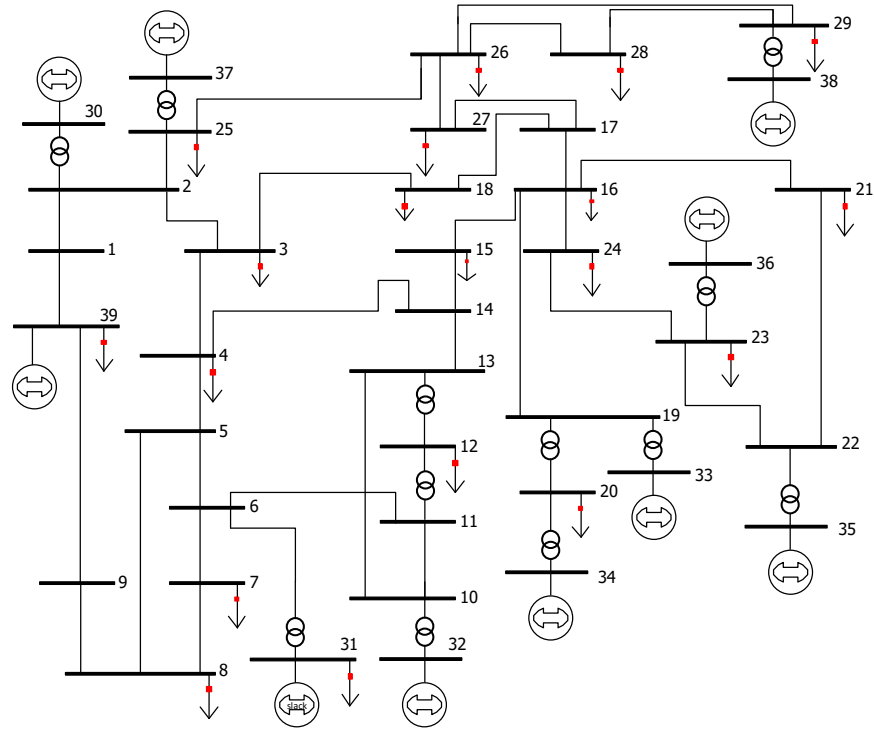

Fig. 1. Single-line diagram of IEEE 39-bus test system
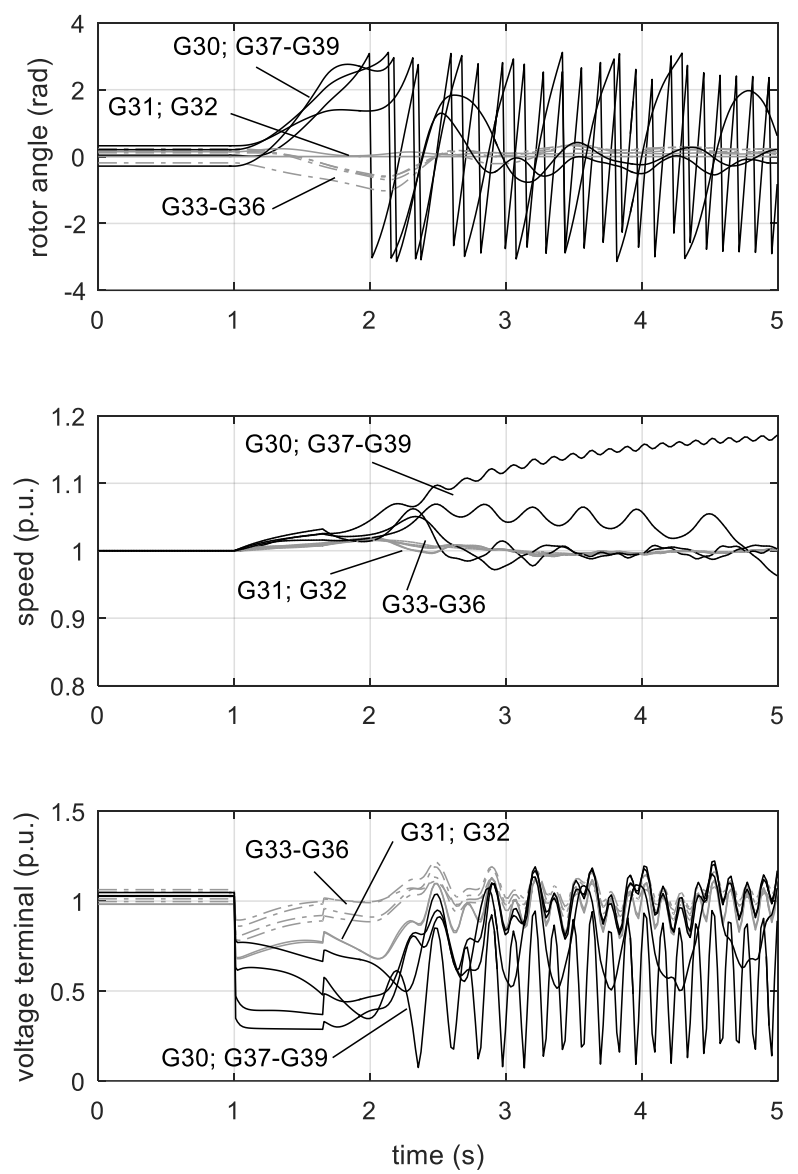

Fig. 2. Case Study 1: IEEE 39-bus test system: Electrical behavior without islanding

prevention constraint included in the strategy (Section 3.2), these weak buses will be assigned into individual islands in such a way to be as close as possible to the reactive power sources (i.e., the generators for this test system). This is 


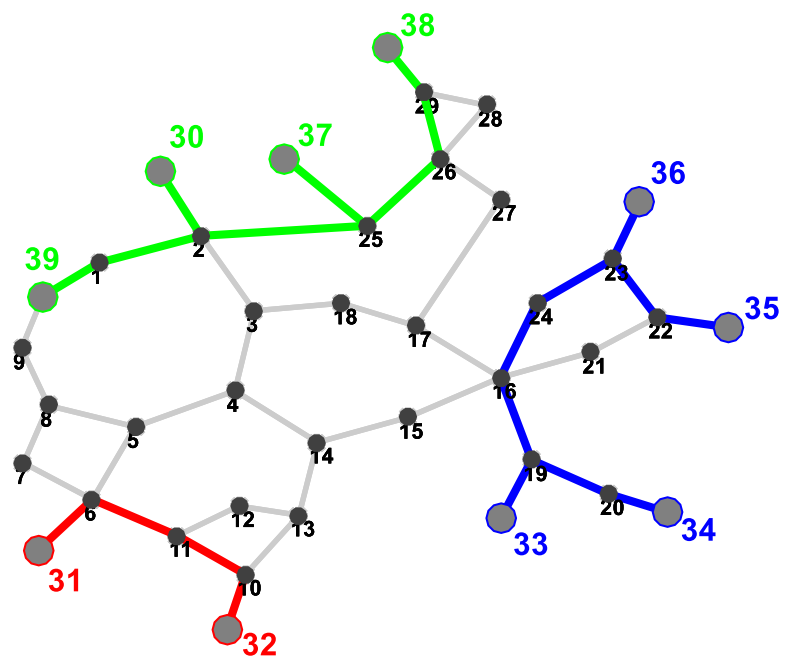

Fig. 3. Case Study 1: Preprossesing procedure on IEEE 39-bus test system

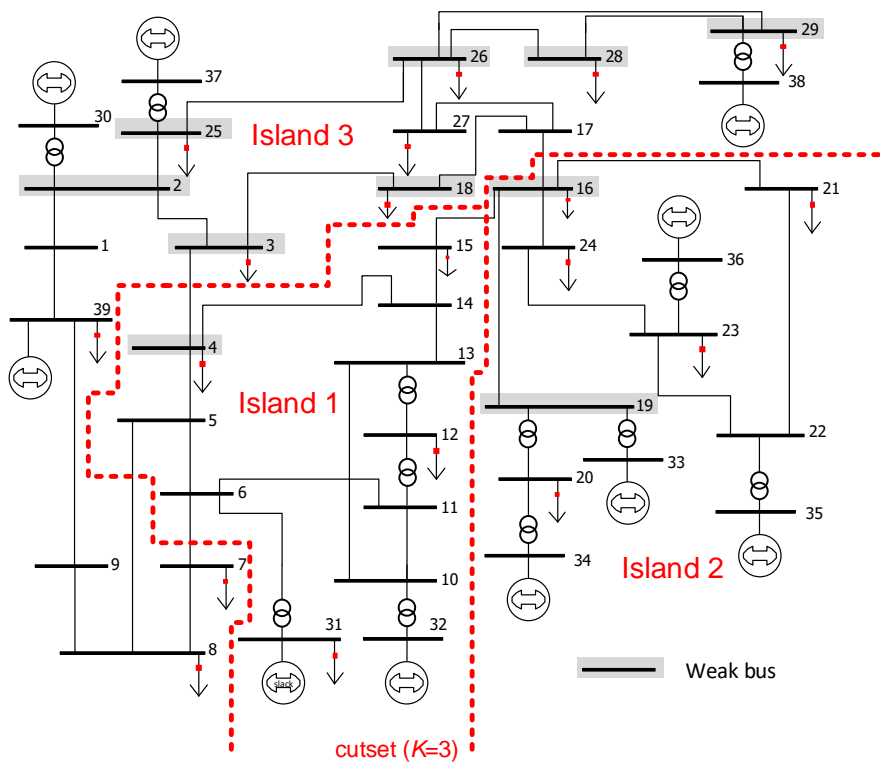

Fig. 4. Case Study 1: IEEE 39-bus test system with optimal islanding solution

TABLE I

EXACT ISLANDING SOLUTION FOR THE IEEE 39-BUS TEST SYSTEM

\begin{tabular}{|c|c|c|c|}
\hline $\begin{array}{c}\text { No. of } \\
\text { Islands }(K)\end{array}$ & Cutset & $\begin{array}{c}\text { Cut } \\
(\boldsymbol{M W})\end{array}$ & $\begin{array}{c}\text { Time } \\
(\boldsymbol{s})\end{array}$ \\
\hline 3 & $3-4,5-8,6-7,15-16,16-17$ & 966.058 & 0.033 \\
\hline
\end{tabular}

TABLE II

LOAD AND GENERATION WITHIN EACH IsLAND FoR THE IEEE 39-BUS TEST SYSTEM SPLIT INTO THREE ISLANDS $(K=3)$

\begin{tabular}{|c|c|c|c|c|c|c|}
\hline \multirow{2}{*}{$\begin{array}{c}\text { Island } \\
\text { number } \\
(k)\end{array}$} & \multirow{2}{*}{$\begin{array}{c}\text { EPload } \\
(M W)\end{array}$} & \multirow{2}{*}{$\begin{array}{l}\text { ¿Qload } \\
(M V A r)\end{array}$} & \multirow{2}{*}{$\begin{array}{c}\text { EPgen } \\
(M W)\end{array}$} & \multirow{2}{*}{$\begin{array}{l}\Sigma Q g e n \\
(M V A r)\end{array}$} & \multicolumn{2}{|c|}{$\begin{array}{c}\text { Generation } \\
\text { capacity }\end{array}$} \\
\hline & & & & & $\boldsymbol{P}_{k}$ & $Q_{k}$ \\
\hline 1 & 836.7 & 429.6 & 1169.9 & 403.4 & 1670 & 727.9 \\
\hline 2 & 3473.3 & 736.6 & 2620 & 257.7 & 4036 & 1117.1 \\
\hline 3 & 1787.1 & 242.9 & 2350 & 589.3 & 3340 & 1455.9 \\
\hline
\end{tabular}

achieved by calculating the electrical distance between each weak bus and all the generators in the system.

The implementation of the proposed ICI algorithm identifies the optimal solution (for minimal power-flow disruption) to open the lines 3-4, 5-8, 7-8, 15-16 and 16-17
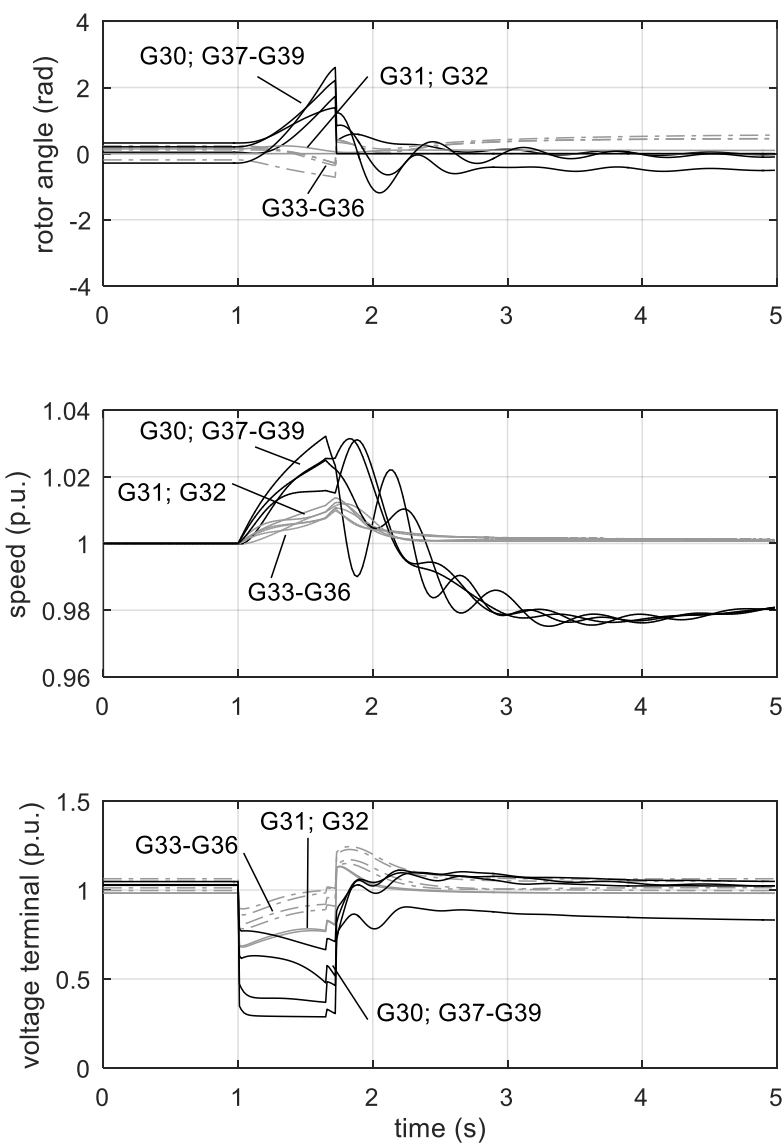

Fig. 5. Case Study 1: IEEE 39-bus test system: Electrical behavior with islanding

TABLE III

ASSIGNMENT OF WEAKEST BUSES INTO ISLANDS FOR THE IEEE 39-BUS TEST SYSTEM

\begin{tabular}{|c|c|c|}
\hline Weak bus & Island assigned & Closest reactive power source \\
\hline $2,3,18,26$ & 3 & $\mathrm{G} 30$ \\
\hline 4 & 1 & $\mathrm{G} 32$ \\
\hline 16 & 2 & $\mathrm{G} 35$ \\
\hline 19 & 2 & $\mathrm{G} 33$ \\
\hline 25 & 3 & $\mathrm{G} 37$ \\
\hline 28,29 & 3 & $\mathrm{G} 38$ \\
\hline
\end{tabular}

(red dotted line in Fig. 4). The solution was found in approximately $0.033 \mathrm{~s}$ (Table I). Hence, islanding was undertaken at $t=1.733 \mathrm{~s}$ (without considering the delay in communications and the actual operation of the CBs). Fig. 5 shows the swing curves of the generators after the solution is applied. As it can be seen, three stable groups are created. Moreover, the frequencies of Islands 1-3 are 1.001 p.u., 1.001 p.u., and 0.981 p.u. respectively (Fig. 5). Voltages also recover close to their nominal values (Fig. 5). Since the system separation successfully recovers the frequency and the voltages of the islands within admissible limits, it can be concluded that the proposed extended ICI algorithm split the system in a controlled manner, preventing the total blackout.

The load and generation (i.e., active and reactive power) in each island, as well as the generation capacity of each island are presented in Table II. As it can be noticed, the ICI 


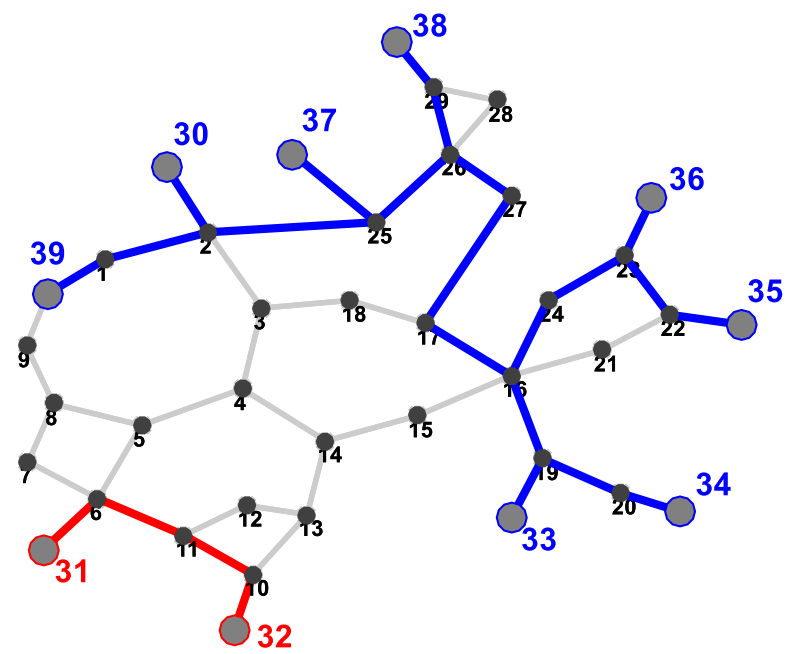

Fig. 6. Case Study 2: Preprossesing procedure on IEEE 39-bus test system

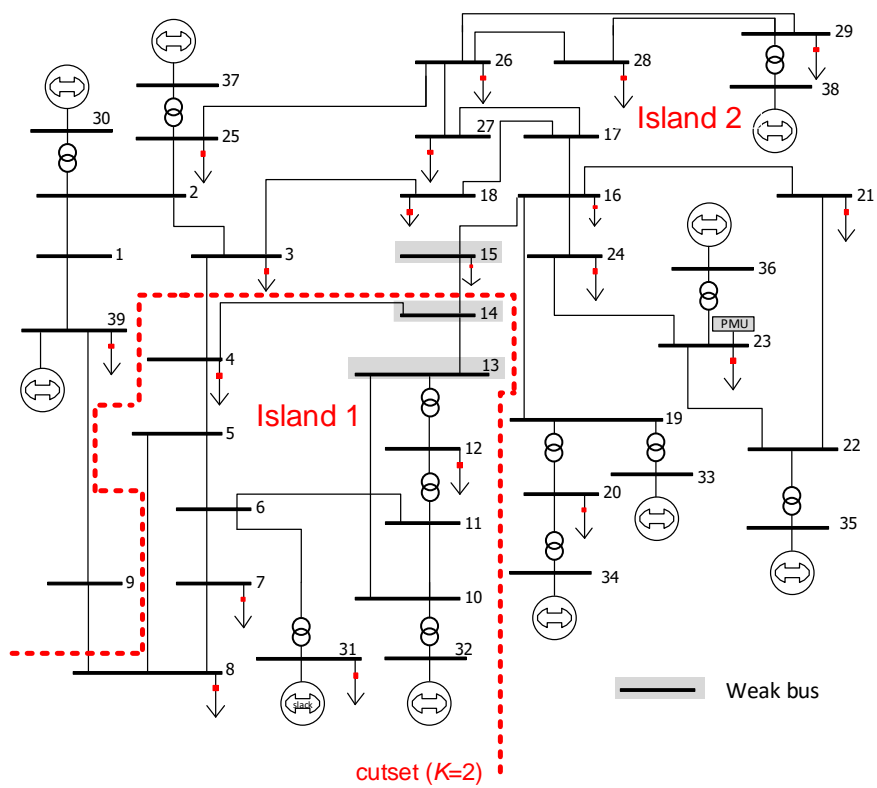

Fig. 7. Case Study 2: IEEE 39-bus test system with optimal islanding solution

TABLE IV

EXACT ISLANDING SOLUTION FOR THE IEEE 39-BUS TEST SySTEM

\begin{tabular}{|c|c|c|c|}
\hline $\begin{array}{c}\text { No. of } \\
\text { Islands }(K)\end{array}$ & Cutset & $\begin{array}{c}\text { Cut } \\
(\boldsymbol{M W})\end{array}$ & $\begin{array}{c}\text { Time } \\
(\boldsymbol{s})\end{array}$ \\
\hline 2 & $3-4,8-9,14-15$ & 152.5826 & 0.041 \\
\hline
\end{tabular}

TABLE V

LOAD AND GENERATION WITHIN EACH ISLAND FOR THE IEEE 39-BUS TEST SYSTEM SPLIT INTO TWO ISLANDS $(K=2)$

\begin{tabular}{|c|c|c|c|c|c|c|}
\hline \multirow{2}{*}{$\begin{array}{c}\text { Island } \\
\text { number } \\
(k)\end{array}$} & \multirow{2}{*}{$\begin{array}{c}\text { EPload } \\
(M W)\end{array}$} & \multirow{2}{*}{$\begin{array}{l}\Sigma Q \text { Qload } \\
(M V A r)\end{array}$} & \multirow{2}{*}{$\begin{array}{c}\text { EPgen } \\
(M W)\end{array}$} & \multirow{2}{*}{$\begin{array}{l}\Sigma Q g e n \\
(M V A r)\end{array}$} & \multicolumn{2}{|c|}{$\begin{array}{c}\text { Generation } \\
\text { capacity }(M W)\end{array}$} \\
\hline & & & & & $\boldsymbol{P}_{k}$ & $Q_{k}$ \\
\hline 1 & 1272.5 & 536.6 & 1169.9 & 403.4 & 1670 & 727.9 \\
\hline 2 & 4824.6 & 872.5 & 4970 & 847.0 & 7376 & 2573.0 \\
\hline
\end{tabular}

TABLE VI

ASSIGNMENT OF WEAKEST BUSES INTO ISLANDS FOR THE IEEE 39-BUS TEST SYSTEM

\begin{tabular}{|c|c|c|}
\hline Weak bus & Island assigned & Closest reactive power source \\
\hline 13,14 & 1 & $\mathrm{G} 32$ \\
\hline 15 & 2 & $\mathrm{G} 35$ \\
\hline
\end{tabular}

algorithm has guaranteed sufficient generation capacity to match the active and reactive power demand in each island (Section 3.1). Moreover, it is important to understand that the proposed ICI algorithm has minimized the electrical distance of each weak bus from its closest generator. For example, the weak bus 3 has been assigned to Island 3 and not to Island 1 because it is electrically closer to G30 (0.0303 p.u.) rather than the G31 (0.0470 p.u.) (Table III). The above adjustments have further improved the transient stability of the formed islands.

\subsection{Case Study 2}

Testing case description: At time $t=1 \mathrm{~s}$, a three phase to ground fault occurs at bus 14 and is cleared $0.55 \mathrm{~s}$ later. Shortly after the fault, a group of 2 generators swing together and the rest ones swing apart. Two groups are created: $\{\mathrm{G} 31$, G32 $\}$ and \{G30, G33, G34, G35, G36, G37, G38, G39\}. Moreover, the generator speeds are increased, while the generator terminal voltages are significantly low. Therefore, it can be concluded that the system needs to be split if theblackout is to be avoided. Here, the necessity to split the system is considered to be at $2.0 \mathrm{~s}$. Hence, considering the information of the system (power flow and topology) at $t=$ $2.0 \mathrm{~s}$, the extended MILP ICI algorithm is used to find the optimal splitting solution.

Fig. 6 shows the trees found by the preprocessing procedure for each coherent group. As mentioned above, the nodes of these trees will be served as an additional constraint to the MILP formulation which will contribute to the reduction of its search space. Fig. 7 illustrates the weakest buses (shaded) identified by the execution of the proposed extended ICI algorithm (i.e., bus 13, 14 and 15), as well as the optimal islanding solution determined. The information about the splitting strategy found, the value of the cut and the execution time are presented in Table IV. The post-islanding behavior of the islands, which is quite similar to the one of the previous case study, highlights that the proposed ICI and restoration scheme successfully prevents the power system blackout.

Table V and VI present the impact of including the new voltage stability constraints in the proposed strategy. As it can be seen, the proposed ICI algorithm has guaranteed sufficient generation capacity (active and reactive power) to match the load consumption within each island. At the same time, it has assigned the weakest buses to the islands in such a way to be as close as possible to the generators, reducing the possibility of voltage collapse.

\section{Conclusion}

In this paper an existing MILP ICI algorithm that directly determines an islanding solution with minimal power-flow disruption for any given number of islands is extended to consider voltage stability constraints. The new constraints deal with sufficient generation capacity (active and reactive power) and reduced possibility of voltage collapse within each created island. The effectiveness of the proposed extended ICI algorithm is tested using a dynamic model of the IEEE 39-bus test system. The simulation results have demonstrated its ability to meet the requirement of real-time 
controlled islanding, while further improving the transient stability of the formed islands.

\section{Acknowledgements}

This work has been supported by the European Union's Horizon 2020 research and innovation programme under grant agreement No 739551 (KIOS CoE) and from the Government of the Republic of Cyprus through the Directorate General for European Programmes, Coordination and Development.

\section{$7 \quad$ List of Variables}

\begin{tabular}{|c|c|}
\hline$B$ & Susceptance matrix \\
\hline $\mathcal{E}, \mathcal{E}_{k}$ & Set of edges in system $\mathcal{G}$ and in island $\mathcal{G}_{k}$ \\
\hline $\mathcal{E}_{c}$ & Set of edges in system $\mathcal{G}$ that cannot be disconnected \\
\hline $\mathcal{E}_{s}$ & Cutset \\
\hline$e_{i, j}$ & Edge from node $i$ to node $j$ \\
\hline FVSI & Fast Voltage Stability Index \\
\hline $\mathcal{G}, \mathcal{G}_{k}$ & System and $k$-th island undirected graphs \\
\hline$h, k$ & Island $\mathcal{G}_{k}$ indexes \\
\hline$K$ & Number of islands \\
\hline$M$ & Minimum number of nodes constant in island $\mathcal{G}_{k}$ \\
\hline$m$ & Number of generators in system $\mathcal{G}$ \\
\hline$n$ & Number of nodes in system $\mathcal{G}$ \\
\hline$n_{k}^{\text {gen }}, n_{k}^{\text {load }}$ & Number of generator and load nodes in island $\mathcal{G}_{k}$ \\
\hline$P_{i, j}$ & Power flow from node $i$ to node $j$ \\
\hline$P_{k}, Q_{k}$ & Active and reactive generation capacity in island $\mathcal{G}_{k}$ \\
\hline $\begin{array}{l}\operatorname{Pgen}_{i, k}^{\min }, \\
\operatorname{Pgen}_{i, k}^{\max }\end{array}$ & $\begin{array}{l}\text { Minimum and maximum active power generation of } \\
\text { node } i \text { in island } \mathcal{G}_{k}\end{array}$ \\
\hline $\begin{array}{l}\operatorname{Pload}_{i, k} \\
\operatorname{Qload}_{i, k}\end{array}$ & $\begin{array}{l}\text { Active and reactive power consumption of node } i \text { in } \\
\text { island } \mathcal{G}_{k}\end{array}$ \\
\hline $\begin{array}{l}Q g e n_{i, k}^{\min } \\
\operatorname{Qgen}_{i, k}^{\max }\end{array}$ & $\begin{array}{l}\text { Minimum and maximum reactive power generation of } \\
\text { node } i \text { in island } \mathcal{G}_{k}\end{array}$ \\
\hline$Q_{r}$ & Reactive power at the receiving end \\
\hline$r_{i, j}$ & Electrical distance between bus $i$ and bus $j$ \\
\hline $\mathcal{T}_{k}$ & $\begin{array}{l}\text { Minimum spanning tree connecting all nodes in } \mathcal{V}_{k}^{\text {gen }} \text { of } \\
\text { island } \mathcal{G}_{k}\end{array}$ \\
\hline $\mathcal{V}, \mathcal{V}_{k}$ & Set of nodes in system $\mathcal{G}$ and in island $\mathcal{G}_{k}$ \\
\hline $\mathcal{V}^{\text {gen }}, \mathcal{L}_{k}^{\text {gen }}$ & Set of generator nodes in system $\mathcal{G}$ and in island $\mathcal{G}_{k}$ \\
\hline $\mathcal{V}_{k}^{T}, \mathcal{E}_{k}^{T}$ & Node set and edge set for $\mathcal{T}_{k}$ \\
\hline $\mathcal{V}_{k}^{\text {load }}$ & Set of load nodes in island $\mathcal{G}_{k}$ \\
\hline$V_{s}$ & Sending end voltage \\
\hline$v_{i}$ & Node $i$ in system $\mathcal{G}$ \\
\hline $\mathcal{W}, w_{i, j}$ & $\begin{array}{l}\text { Edge weights set and weight of edge from node } i \text { to } \\
\text { node } j\end{array}$ \\
\hline$X$ & Line reactance \\
\hline$Z$ & Line impedance \\
\hline$z_{i, j}$ & Optimization decision variable \\
\hline
\end{tabular}

\section{References}

[1] Kundur, P., Taylor, C.W.: 'Blackout experiences and lessons, best practices for system dynamic performance, and the role of new technologies', IEEE Task Force Report 2007.

[2] Adibi, M.M., Kafka, R.J.: 'Power system restoration issues', IEEE Computer Applications in Power, 1991, 4, (2), pp. 19-24.

[3] Knight, U.G.: 'Power System in Emergencies', 2001.

[4] Ding, L., Gonzalez-Longatt, F., Wall, P., Terzija, V.: 'Two-step spectral clustering controlled islanding algorithm', IEEE Transactions on Power Systems, 2013, 28, (1), pp. 75-84.

[5] Demetriou, P., Kyriacou, A., Kyriakides, E., Panayiotou, C.: 'Applying exact MILP formulation for controlled islanding of power systems', 51st International Universities Power Engineering Conference, Coimbra, Portugal, Jul. 2016.

[6] Demetriou, P., Kyriacou, A., Kyriakides, E., Panayiotou, C.: 'System splitting strategy considering power system restoration systems', IEEE PowerTech 2017, Manchester, UK, Jul. 2017

[7] Demetriou, P., Asprou, M., Kyriakides, E.: 'A real-time controlled islanding and restoration scheme based on estimated states', IEEE Transactions on Power Systems, 2019, 34, (1), pp. 606-615.

[8] Demetriou, P., Kyriacou, A., Kyriakides, E., Panayiotou, C.: 'Intentional Controlled Islanding of Power Systems Equipped With Battery Energy Storage Systems', IEEE PowerTech 2019, Milano, Italy, Jun. 2019.

[9] Bondy, J.A., Murty, U.S.R.: 'Graph Theory' (Springer, 2nd edn. 2008).

[10] Kyriacou, A,. Timotheou, S., Michaelides, M., Panayiotou, C., Polycarpou, M,: 'Partitioning of intelligent buildings for distributed contaminant detection and isolation', IEEE Transactions on Emerging Topics in Computational Intelligence, 2017, 1, (2), pp. 72-86.

[11] Musirin, I., Abdul Rahman, T.K.: 'Novel fast voltage stability index (FVSI) for voltage stability analysis in power transmission system', Research and Development, Student Conference on SCOReD, Malaysia, Jul. 2002.

[12] Cotilla-Sanchez, E., et al., 'Multi-Attribute Partitioning of Power Networks Based on Electrical Distance', IEEE Transaction on Power Systems, 2013, 28, (4), pp. 4979-4987.

[13] Demetriou, P., Asprou, M., Quiros-Tortos, J., Kyriakides, E.: 'Dynamic IEEE test systems for transient analysis', IEEE Systems Journal, 2017, 11, (4), pp. 2108-2117. 